\title{
BMJ Open Nutrition screening and assessment tools for patients with cancer and survivors of cancer: a systematic review protocol
}

\author{
Marianne Boll Kristensen (D) , ${ }^{1,2}$ Irene Wessel (D) , Kim Skov Ustrup (D) , ${ }^{1}$ \\ Karin B Dieperink (D) , ${ }^{4,5}$ Ann-Dorthe Zwisler (D) ,2 Anne Marie Beck (D) 1,6
}

To cite: Kristensen MB, Wessel I, Ustrup KS, et al. Nutrition screening and assessment tools for patients with cancer and survivors of cancer: a systematic review protocol. BMJ Open 2020;10:e037844. doi:10.1136/ bmjopen-2020-037844

- Prepublication history for this paper is available online. To view these files, please visit the journal online (http://dx.doi. org/10.1136/bmjopen-2020037844).

Received 18 February 2020 Revised 17 August 2020 Accepted 18 August 2020
Check for updates

(C) Author(s) (or their employer(s)) 2020. Re-use permitted under CC BY-NC. No commercial re-use. See rights and permissions. Published by BMJ.

For numbered affiliations see end of article.

Correspondence to Marianne Boll Kristensen; mabk@kp.dk

\section{ABSTRACT}

Introduction Nutritional challenges are common consequences of cancer, and they do not only occur in the hospital setting. They are also frequent after completion of treatment, and nutritional interventions in communitybased post-treatment rehabilitation services are important. The first step towards initiating any nutritional intervention is to identify the individual in need hereof, but evidence is limited on the applicability of different nutrition screening and assessment tools in the post-treatment rehabilitation services. The aim is to systematically review and identify nutrition screening and assessment tools appropriate for use in patients with cancer and survivors of cancer in hospital or community-based healthcare settings. Methods and analysis In this systematic review, the electronic databases PubMed, CINAHL Complete and Embase were searched systematically using comprehensive search strategies. Primary searches were carried out in August 2018 with updated searches performed in November 2019. Clinicaltrials.gov and PROSPERO International Prospective Register of Systematic Reviews will be searched for additional relevant studies. Studies will be included if they validate a nutrition screening or assessment tool in adult patients with cancer or survivors of cancer. No restriction on publication date will be applied, and full-text articles in English, Danish, Norwegian and Swedish are eligible for inclusion. Two reviewers will independently conduct screening of search results, study selection, data extraction and quality assessment. Data will be synthesised narratively.

Ethics and dissemination No ethical approval is required. Results will be reported in accordance with the Preferred Reporting Items for Systematic Reviews and Meta-Analyses statement and published in an international peer-reviewed journal. Furthermore, results will be presented in relevant research and clinical fora to facilitate transfer of results to clinical practice in benefit of patients. PROSPERO registration number CRD42018096678.

\section{INTRODUCTION}

Nutritional challenges are common consequences of cancer, and their occurrence is not restricted to the hospital setting. ${ }^{12}$ Cancer and cancer treatment may affect nutritional
Strengths and limitations of this study

- The systematic review protocol has been developed in accordance with the Preferred Reporting Items for Systematic review and Meta-Analysis Protocols guidelines.

- A comprehensive literature search strategy has been developed to retrieve a broad spectrum of relevant studies.

- Excluding other publication types than full text articles may impose a risk of relevant grey literature being excluded.

intake and requirements, ${ }^{13}$ and the high risk of cancer-related malnutrition has been documented in several groups of patients with cancer $^{4-9}$ and survivors of cancer. ${ }^{10}$ Nutrition impact symptoms affecting dietary intake, nutritional status and quality of life are frequent, ${ }^{10-13}$ and may occur or persist years after completion of treatment. ${ }^{10} 14$ Hence, nutritional challenges are not only topics of concern in the hospital setting during cancer treatment but also in the community-based post-treatment rehabilitation services. The nature of the nutritional challenges may vary across settings. In the hospital setting where patients with cancer are in the acute phase of their trajectory, tumour-related metabolic derangements and systemic inflammation contribute greatly to cancer-related malnutrition. ${ }^{3}$ In the posttreatment phase, nutritional challenges are often caused by nutrition impact symptoms and other late effects ${ }^{15}$ rather than by a metabolic stress response.

For several cancer types, increasing incidence rates combined with increased survival ${ }^{16}$ have led to an increase in the population of survivors of cancer and thereby an increased demand for appropriate community-based 
post-treatment rehabilitation services including management of nutritional challenges.

Nutritional interventions can improve nutritional status and quality of life in some patients with cancer ${ }^{17} 18$ and survivors of cancer, ${ }^{19}$ but initiation of any nutritional intervention requires a systematic identification of the individual patient who requires and will benefit from the intervention. The European Society for Clinical Nutrition and Metabolism recommends that nutrition screening is performed at cancer diagnosis and repeated regularly depending on the clinical situation, ${ }^{1}$ and that any nutritional intervention is initiated with a nutritional assessment of among others dietary intake and nutrition impact symptoms. ${ }^{1}$ Yet, there is no consensus on which tools to use in this nutrition screening or assessment in the different healthcare settings. Several tools have been developed to screen for and assess nutritional risk, nutritional status and nutrition impact symptoms, ${ }^{1} 2021$ but many of these tools have primarily been validated in patients with cancer during treatment. Less is known on their ability to assess post-treatment nutritional challenges and to identify survivors of cancer who will benefit from post-treatment nutritional interventions. Since the nature of nutritional challenges may vary across settings, so may the applicability of different nutrition screening and assessment tools. Furthermore, nutrition screening and assessment tools may have been designed for different purposes, for example, identify nutritional status, identify need for nutritional interventions or predict effect of nutritional interventions. ${ }^{22}$ An overview of the different nutrition screening and assessment tools for patients with cancer and survivors of cancer and the tools' applicability in the different healthcare settings could inform healthcare professionals in the selection of tools for their clinical practice. Hence, this systematic review seeks to answer the following research question: "Which nutrition screening and assessment tools are appropriate for systematic nutrition screening and assessment in patients with cancer and survivors of cancer in hospital or community-based healthcare settings?'.

\section{Study objectives}

The objectives of the study are:

- To identify nutrition screening and assessment tools validated in patients with cancer and/or survivors of cancer.

- To evaluate the following for each identified tool:

- Purpose of the tool: is the tool validated for assessment of nutritional status as defined by the authors of the given study, nutritional risk, nutrition impact symptoms, to identify patients who will benefit from nutritional intervention and so onor other purposes? Benefit from nutritional interventions can be defined as a clinically relevant improvement in clinical outcome following nutritional interventions in patients with cancer/survivors of cancer who have been categorised as being malnourished/at nutritional risk according to the tested tool.
- Patient group: which patient groups according to cancer diagnoses and treatment modality has the tool been validated in?

- Setting: is the tool validated in patients with cancer during treatment or survivors of cancer after completion of treatment?

- Administration: is the tool supposed to be administered or filled out by health professionals or patients?

- Validation: how was the tool validated? (content validity or other domains recommended by COnsensus-based Standards for the selection of health Measurement INstruments (COSMIN), ${ }^{23}$ predictive values compared with a gold standard or a given clinically relevant outcome, etc).

- To discuss whether the identified tools can be recommended for use in systematic nutrition screening and assessment in patients with cancer and survivors of cancer, and at which time point of the cancer trajectory the tool will be relevant to use.

\section{METHODS AND ANALYSIS}

This systematic review protocol has been developed in accordance with the Preferred Reporting Items for Systematic review and Meta-Analysis Protocols statement. ${ }^{24}$

\section{Eligibility criteria}

Inclusion and exclusion criteria based on population, exposure, comparators and outcome are summarised in table 1 .

\section{Population}

The population of interest includes adult patients with cancer and survivors of cancer (18+ years).

No restriction on cancer type is applied, and studies are included regardless of whether they focus on one specific group of patients with cancer/survivors of cancer or on a heterogeneous group of patients with cancer/survivors of cancer. Studies including patients with cancer/survivors of cancer as a part of a heterogeneous patient population will only be included, if data for the subgroup of patients with cancer/survivors of cancer are presented separately. In that case, only data regarding the cancer subgroup will be extracted.

\section{Exposure}

Studies will be included if they test the validity of a given nutrition screening or assessment tools. To assess the eligibility of the studies, the following definition of nutrition screening and assessment tools will be used: tools (including patient reported outcome measures) that combine more than one variable related to nutritional intake, nutritional risk, nutritional status ${ }^{25}$ and nutrition impact symptoms (as described under the Search strategies section) to yield an overall result. Hence, studies assessing single variables, for example, body weight, will not be included, while a study that uses a combination of, for example, body weight and dietary intake to categorise participants according to nutritional status or risk 
Table 1 Inclusion and exclusion criteria

\begin{tabular}{lll} 
& Inclusion criteria & Exclusion criteria \\
\hline Population & $\begin{array}{l}\text { Adult patients with cancer and survivors } \\
\text { of cancer (18+ years) }\end{array}$ & \\
Exposure & Nutrition screening or assessment with $\quad$ Nutrition screening or assessment with \\
& a tool that assesses nutritional status, tools that includes invasive measures \\
& nutritional risk, nutrition impact symptoms (eg, blood samples) \\
& or identifies individuals who needs or will \\
& benefit from nutritional interventions using \\
& more than one variable to yield an overall \\
& result
\end{tabular}

\begin{tabular}{|c|c|c|}
\hline Comparators & $\begin{array}{l}\text { Gold standard (defined by the authors of } \\
\text { the given study) or none }\end{array}$ & \\
\hline Outcomes & $\begin{array}{l}\text { Studies testing the validity of a tool } \\
\text { Validity, predictive value, sensitivity, } \\
\text { specificity, accuracy, internal consistency, } \\
\text { reliability, measurement error, content } \\
\text { validity, structural validity, hypotheses } \\
\text { testing, cross-cultural validity, criterion } \\
\text { validity, responsiveness, prognostic value }\end{array}$ & $\begin{array}{l}\text { Studies merely performing linguistic } \\
\text { validation of a given tool }\end{array}$ \\
\hline \multicolumn{3}{|l|}{ Types of studies } \\
\hline Study design & $\begin{array}{l}\text { Clinical studies (controlled and } \\
\text { uncontrolled clinical trials, observational } \\
\text { studies), systematic reviews }\end{array}$ & Case studies, non-systematic reviews \\
\hline Publication type & Full text articles & \\
\hline
\end{tabular}

can be eligible. The definition of nutritional status or risk may vary across included studies, and studies who fulfil remaining eligibility criteria will not be excluded based on their definition of nutritional status. Since blood samples and other biochemical tests are not readily available in all healthcare settings, the tools must comprise non-invasive measurements. Hence, tools including blood samples and other biochemical tests will be excluded.

\section{Comparators}

Comparators can be a gold standard defined by the authors of the given study or none. The gold standard may vary between studies depending on the type of tool being assessed, and an example of a gold standard could be another validated assessment tool or a clinical test. Studies with no comparator may include studies assessing internal consistency, cross-cultural validity and other domains where a comparator is not needed.

\section{Outcomes}

Studies will be included if they test the validity of a tool using any of the following outcomes: validity, predictive value, sensitivity, specificity, accuracy, internal consistency, reliability, measurement error, content validity, structural validity, hypotheses testing, cross-cultural validity, criterion validity, responsiveness, prognostic value.

The validity can be tested against a gold standard or a given clinically relevant outcome related to a nutritional intervention, for example, body weight, benefit from nutritional intervention, etc. Benefit from nutritional interventions is defined as clinically relevant improvements in clinical outcome following nutritional interventions in patients with cancer/survivors of cancer categorised as being malnourished/at nutritional risk with the given tool. Studies merely performing linguistic validation of translated tools will not be included. However, a note will be added to these studies, and they may be included in the discussion of the worldwide availability of a given tool.

Types of studies to be included

Clinical studies (controlled and uncontrolled clinical trials, observational studies) and systematic reviews will be considered for inclusion, while case studies and nonsystematic reviews will be excluded. No restriction on publication date will be applied, and full-text articles in English, Danish, Swedish, Norwegian will be considered for inclusion. The decision to only include full text articles may impose a risk of grey literature identified in Clinicaltrials.gov and PROSPERO International Prospective Register of Systematic Reviews ${ }^{26}$ being excluded. Yet, we find that full text access is necessary to make a comprehensive evaluation of whether a study meets the other inclusion criteria.

\section{Search strategies}

The primary searches were performed in the following electronic databases: PubMed, CINAHL Complete and 
Table 2 Search terms included in the search strategies for the electronic databases PubMed, CINAHL Complete and Embase

\begin{tabular}{ll}
\hline Population & Cancer survivor(s), cancer patient(s), neoplasms \\
\hline Exposure & nutrition(al) assessment, nutrition(al) screening \\
& OR needs assessment, self-reported outcome(s), patient reported outcome(s), patient reported outcome \\
& measures, screening, assessment, tool(s), instrument(s), questionnaire(s), surveys and questionnaires \\
& AND nutrition(al), nutritional status, malnutrition, nutrition therapy, nutrition impact symptom(s), feeding \\
& and eating disorders, eating difficulty/difficulties, eating problem(s), appetite, anorexia, xerostomia, dry \\
& mouth, mouth dryness, hyposalivation, dysgeusia, altered taste, taste alteration(s), distorted taste, taste \\
& distortion(s), taste change(s), abdominal pain, dyspepsia, mouth pain, oral pain, sore mouth, mouth \\
& soreness, mucositis, stomatitis, dysphagia, swallowing difficulty/difficulties, swallowing disorder(s), \\
& deglutition disorder(s), oesophageal motility disorders, chewing difficulty/difficulties, dental problem(s), \\
& poor dentition, digestive problem(s), digestive symptom(s), gastrointestinal symptom(s), nausea, \\
& vomit(ing), vomitus, constipation, constipated, diarrhoea, flatulence, meteorism, bloating, bloatedness, \\
bloated, weight, body weight, body weight changes, public eating
\end{tabular}

Embase. The searches were not date restricted and they were based on combinations of the search terms showed in table 2. The exact search strategy for one of the databases is provided in online supplemental file 1. Furthermore, Clinicaltrials.gov, PROSPERO International Prospective Register of Systematic Reviews, ${ }^{26}$ Cochrane Library and reference lists of included articles will be searched to identify additional relevant studies published as full-text articles.

The search strategies for the electronic databases were developed with support from an information specialist. Search terms related to the exposure were selected with inspiration from The European Organization for Research and Treatment of Cancer's (EORTC) quality of life questionnaire, QLQ-C30 $0^{27}$ and relevant diagnosis specific modules for different types of cancer. ${ }^{28-38}$ The search terms are based on items related to nutritional intake, nutritional status and nutrition impact symptoms. These nutrition impact symptoms include eating difficulties, anorexia, xerostomia, dysgeusia, abdominal pain, dyspepsia, oral pain, mucositis, stomatitis, dysphagia, dental problems, nausea, vomiting, constipation, diarrhoea, flatulence, meteorism and body weight changes.

Search terms related to outcomes were selected to identify studies that either test validity as accuracy, sensitivity, specificity or predictive values compared with a gold standard or a given clinically relevant outcome or assesses the domains recommended by $\operatorname{COSMIN}^{23}$ for assessment of methodological quality of studies on measurement properties (internal consistency, reliability, measurement error, content validity, structural validity, hypotheses testing, cross-cultural validity, criterion validity and responsiveness).

The protocol was registered at PROSPERO International Prospective Register of Systematic Reviews ${ }^{26}$ before the primary searches were carried out in August 2018. Updated searches using the same search strategy were performed in November 2019.

\section{Study selection}

The Covidence ${ }^{39}$ software tool for systematic reviews will be used in the selection and management of articles. Duplicates will be removed after transferring searches to Covidence. Removal of duplicates will be documented in a flow chart of the selection process.

The titles and abstracts of all identified articles will be screened for relevance by two authors (MBK+either AMB, IW or KSU) independently. All articles with irrelevant titles and/or abstracts will be excluded. Two authors (MBK+either AMB, IW or KSU) will independently inspect the full text of the remaining articles and decide on their eligibility for inclusion using the inclusion and exclusion criteria. Reasons for exclusion of articles in the full text screening will be documented, and a flow chart of the selection process will be developed. A list of references excluded in the full text screening will be available on request.

Possible disagreement between the two authors in the selection process will be solved through discussion or by involving a third independent author. As a pilot test of the study selection process, all four authors who will be involved in the selection of studies (MBK, AMB, IW, KSU) initially screened the same 25 references and potential conflicts were solved by discussion.

\section{Data extraction}

Data extraction will be performed by two authors independently. The following data will be extracted using a customised data extraction form:

- Study information: authors, publication year, source of publication.

- Methods and study design.

- Population: description of study population, setting of recruitment, inclusion criteria, exclusion criteria.

- Exposure: the studied screening or assessment tool(s)

- Outcome: how the validity of the studied screening or assessment tool(s) is assessed.

- Study results. 
- Review of authors conclusions.

- Evaluation of potential bias.

- Funding of the study and reported conflict of interests of the authors.

\section{Risk of bias assessment}

The methodological quality of included papers will be assessed using appropriate checklists according to study type.

Randomised clinical trials are assessed using the Cochrane Risk of Bias Tool. ${ }^{40}$ Observational studies (cohort studies and case-control studies) are assessed using Risk Of Bias In Non-randomized Studies - of Interventions (ROBINS-I), ${ }^{41}$ and diagnostic primary studies will be assessed using the checklist developed by the Quality assessment of Diagnostic Accuracy Studies (QUADAS) Initiative. ${ }^{42}$ Systematic reviews are assessed using the A MeaSurement Tool to Assess systematic Reviews (AMSTAR) 2 checklist. $^{43}$

Studies will not be excluded based on quality issues. However, issues related to the methodological quality will be documented and considered in the analysis and the conclusions of the review. These considerations will be stated explicitly in the presentation of the results.

\section{Strategy for data synthesis}

A summary of extracted data will be presented in tables in the presentation and dissemination of the results. Analysis of data will include comparisons of the different screening and assessment tools with regards to how they are validated, target group and type of tool (patient reported or performed by health professionals). No meta-analyses will be made, and comparisons will be described narratively.

All included articles will be presented in a table with information on authors, publication year, study type, methods and results. Furthermore, identified tools will be presented in tables with information on their purpose, patient group (cancer diagnoses and treatment modality), setting (hospital/during treatment or post-treatment), administration and validation (method, outcome and result). In these tables, tools will be grouped according to their purpose (eg, to assess anorexia) to allow for comparison and to make results targeted and clinically meaningful for clinicians. Discussions on whether identified tools can be recommended for systematic nutrition screening and assessment in patients with cancer and survivors of cancer and on which time point each tool will be relevant to use will be based on results summarised in the tables. Within the given setting, patient group and group of tools according to their purpose, highest recommendations will be given to the tool that have been validated most thoroughly and hence, have been validated across the largest number of domains and in studies with the highest methodological quality.

\section{Analysis of subgroups or subsets}

In addition to the full overview of tools, data will be analysed and presented separately for tools validated in patients with cancer during treatment and survivors of cancer post-treatment, respectively. In these tables, results will be presented according to which cancer diagnoses the given tools have been validated in (eg, heterogenous groups of patients with cancer, patients with head and neck cancer, etc).

\section{Study status}

The protocol was registered within PROSPERO International Prospective Register of Systematic Reviews ${ }^{26}$ in June 2018. Primary searches were carried out in August 2018 and updated searches using the same search strategies were performed in November 2019. Study selection and data extraction is expected to be completed in October 2020.

\section{ETHICS AND DISSEMINATION}

No ethical approval is required for this systematic review, as it is based on published articles, and does not directly include patients. Results will be reported in accordance with the Preferred Reporting Items for Systematic Reviews and Meta-Analyses Statement ${ }^{44}$ and published in an international peer reviewed journal. Results will furthermore be presented at national and international conferences.

\section{PATIENT AND PUBLIC INVOLVEMENT}

No patients or public stakeholders were involved in the development of this systematic review protocol. The results of the systematic review will inform and form the basis of future clinical studies where patients and public stakeholders will be invited to participate in development of study designs and protocols. In this process, patients' perceptions of user-friendliness and relevance of the tools identified in this review will be taken into consideration when selecting nutrition screening and assessment tools to use for inclusion criteria and/or outcome measures.

\section{DISCUSSION}

This systematic review will identify nutrition screening and assessment tools appropriate for use in systematic nutrition screening and assessment in patients with cancer and survivors of cancer. The review will provide health professionals in hospitals and community-based healthcare services with a useful overview that can inform them in their selection of tools.

The scope of the systematic review is broad as it includes different types of tools, tools for all cancer diagnoses and tools validated in both patients with cancer during treatment and survivors of cancer after treatment. It could be argued, that a narrower scope focusing on one setting or one group of patients with cancer would have increased the applicability of review results. However, while cancerrelated health services at hospitals are often highly specialised and diagnosis specific, community-based healthcare services are often aimed at heterogenous groups of cancer 
survivors. ${ }^{45}$ So, for many clinicians in community-based healthcare services, this broad group may represent the target group they see every day in their clinical practice. For these clinicians, a broad overview can be very useful since it will provide a better understanding of generic and cancer diagnosis specific tools, and of how the different tools might supplement each other. Despite the broad scope of the review, results will be presented separately for specific groups of patients with cancer/survivors of cancer allowing health professionals to select between diagnosis-specific and generic tools to improve their clinical practice depending on the target population.

Excluding tools that include blood samples and other invasive measures may lead to exclusion of relevant tools, since blood samples can be good indicators of inflammation and hence, of metabolic derangements during the acute phase of the trajectory. ${ }^{3}$ This exclusion criteria was applied since blood samples are not readily available in all healthcare settings and to ensure that the identified screening tools are brief and inexpensive as the recommendation from The European Society for Clinical Nutrition and Metabolism prescribes. ${ }^{1}$

A strengthening of the evidence on nutrition screening and assessment in patients with cancer and survivors of cancer may contribute to strengthen the implementation of systematic nutrition screening and assessment, and to ensure that patients with cancer with documented needs of nutritional interventions are referred to appropriate services during and after cancer treatment.

\section{Author affiliations}

${ }^{1}$ Department of Nursing and Nutrition, University College Copenhagen, Copenhagen, Denmark

${ }^{2}$ REHPA, The Danish Knowledge Centre for Rehabilitation and Palliative Care, Odense University Hospital, and Department of Clinical Research, University of Southern Denmark, Nyborg, Denmark

${ }^{3}$ Department of Otorhinolaryngology, Head and Neck Surgery \& Audiology,

Rigshospitalet, Copenhagen, Denmark

${ }^{4}$ Research Unit of Oncology, Department of Oncology, Odense University Hospital, Odense, Denmark

${ }^{5}$ Department of Clinical Research, University of Southern Denmark, Odense, Denmark

${ }^{6}$ Dietetics and Clinical Nutrition Research Unit, Herlev and Gentofte Hospital, Herlev, Denmark

Contributors MBK, AMB, IW, KSU, ADZ and KD designed systematic review and developed the review protocol. MBK and AMB drafted the manuscript, which was revised critically by all authors. All authors approved the final version to be published.

Funding This work is externally supported by Innovation Fund Denmark grant number 6171-00009B through the principal researcher's (MBK) enrolment in the public sector industrial PhD programme.

Competing interests None declared.

Patient and public involvement Patients and/or the public were not involved in the design, or conduct, or reporting, or dissemination plans of this research.

Patient consent for publication Not required.

Provenance and peer review Not commissioned; externally peer reviewed.

Open access This is an open access article distributed in accordance with the Creative Commons Attribution Non Commercial (CC BY-NC 4.0) license, which permits others to distribute, remix, adapt, build upon this work non-commercially, and license their derivative works on different terms, provided the original work is properly cited, appropriate credit is given, any changes made indicated, and the use is non-commercial. See: http://creativecommons.org/licenses/by-nc/4.0/.

\section{ORCID iDs}

Marianne Boll Kristensen http://orcid.org/0000-0002-7307-7215

Irene Wessel http://orcid.org/0000-0001-9986-5001

Kim Skov Ustrup http://orcid.org/0000-0002-6865-8007

Karin B Dieperink http://orcid.org/0000-0003-4766-3242

Ann-Dorthe Zwisler http://orcid.org/0000-0002-9241-2090

Anne Marie Beck http://orcid.org/0000-0003-1210-0167

\section{REFERENCES}

1 Arends J, Bachmann P, Baracos V, et al. ESPEN guidelines on nutrition in cancer patients. Clin Nutr 2017;36:11-48.

2 Freijer K, Tan SS, Koopmanschap MA, et al. The economic costs of disease related malnutrition. Clin Nutr 2013;32:136-41.

3 Arends J, Baracos V, Bertz H, et al. ESPEN expert group recommendations for action against cancer-related malnutrition. Clin Nutr 2017;36:1187-96.

4 Kubrak C, Martin L, Gramlich L, et al. Prevalence and prognostic significance of malnutrition in patients with cancers of the head and neck. Clin Nutr 2020;39:901-9.

5 Pressoir M, Desné S, Berchery D, et al. Prevalence, risk factors and clinical implications of malnutrition in French comprehensive cancer centres. Br J Cancer 2010;102:966-71.

6 Fukuda Y, Yamamoto K, Hirao M, et al. Prevalence of malnutrition among gastric cancer patients undergoing gastrectomy and optimal preoperative nutritional support for preventing surgical site infections. Ann Surg Oncol 2015;22 Suppl 3:778-85.

7 Wie G-A, Cho Y-A, Kim S-Y, et al. Prevalence and risk factors of malnutrition among cancer patients according to tumor location and stage in the National cancer center in Korea. Nutrition 2010;26:263-8.

8 Hébuterne X, Lemarié E, Michallet M, et al. Prevalence of malnutrition and current use of nutrition support in patients with cancer. JPEN $J$ Parenter Enteral Nutr 2014;38:196-204.

9 Planas M, Álvarez-Hernández J, León-Sanz M, et al. Prevalence of hospital malnutrition in cancer patients: a sub-analysis of the PREDyCES $\otimes$ study. Support Care Cancer 2016;24:429-35.

10 Crowder SL, Douglas KG, Yanina Pepino M, et al. Nutrition impact symptoms and associated outcomes in post-chemoradiotherapy head and neck cancer survivors: a systematic review. J Cancer Surviv 2018;12:479-94.

11 de Pinho NB, Martucci RB, Rodrigues VD, et al. High prevalence of malnutrition and nutrition impact symptoms in older patients with cancer: results of a Brazilian multicenter study. Cancer 2020;126:156-64.

12 Omlin A, Blum D, Wierecky J, et al. Nutrition impact symptoms in advanced cancer patients: frequency and specific interventions, a case-control study. J Cachexia Sarcopenia Muscle 2013;4:55-61.

13 Tong $H$, Isenring $E$, Yates $P$. The prevalence of nutrition impact symptoms and their relationship to quality of life and clinical outcomes in medical oncology patients. Support Care Cancer 2009;17:83-90.

14 Schandl A, Lagergren J, Johar A, et al. Health-Related quality of life 10 years after oesophageal cancer surgery. Eur J Cancer 2016;69:43-50.

15 Rock CL, Doyle C, Demark-Wahnefried W, et al. Nutrition and physical activity guidelines for cancer survivors. CA Cancer J Clin 2012;62:242-74.

16 Torre LA, Siegel RL, Ward EM, et al. Global Cancer Incidence and Mortality Rates and Trends--An Update. Cancer Epidemiol Biomarkers Prev 2016;25:16-27.

17 Halfdanarson TR, Thordardottir E, West CP, et al. Does dietary counseling improve quality of life in cancer patients? A systematic review and meta-analysis. J Support Oncol 2008;6:234-7.

18 Baldwin C, Spiro A, Ahern R, et al. Oral nutritional interventions in malnourished patients with cancer: a systematic review and metaanalysis. J Natl Cancer Inst 2012;104:371-85.

19 Eades M, Murphy J, Carney S, et al. Effect of an interdisciplinary rehabilitation program on quality of life in patients with head and neck cancer: review of clinical experience. Head Neck 2013;35:343-9.

20 Mendes NP, Barros TAde, Rosa CdeOB, et al. Nutritional screening tools used and validated for cancer patients: a systematic review. Nutr Cancer 2019;71:898-907.

21 Jager-Wittenaar $\mathrm{H}$, Ottery FD. Assessing nutritional status in cancer: role of the Patient-Generated subjective global assessment. Curr Opin Clin Nutr Metab Care 2017;20:322-9. 
22 Elia M, Stratton RJ. An analytic appraisal of nutrition screening tools supported by original data with particular reference to age. Nutrition 2012;28:477-94.

23 Prinsen CAC, Mokkink LB, Bouter LM, et al. COSMIN guideline for systematic reviews of patient-reported outcome measures. Qual Life Res 2018;27:1147-57.

24 Moher D, Shamseer L, Clarke M, et al. Preferred reporting items for systematic review and meta-analysis protocols (PRISMA-P) 2015 statement. Syst Rev 2015;4:1.

25 Cederholm T, Barazzoni R, Austin P, et al. ESPEN guidelines on definitions and terminology of clinical nutrition. Clin Nutr 2017;36:49-64.

26 NHS - National Institute for Health Research. PROSPERO international prospective register of systematic reviews [Internet]. Available: https://www.crd.york.ac.uk/PROSPERO/ [Accessed 16 Jan 2020].

27 Aaronson NK, Ahmedzai S, Bergman B, et al. The European organization for research and treatment of cancer QLQ-C30: a quality-of-life instrument for use in international clinical trials in oncology. J Natl Cancer Inst 1993;85:365-76.

28 Sprangers MA, Groenvold M, Arraras Jl, et al. The European organization for research and treatment of cancer breast cancerspecific quality-of-life questionnaire module: first results from a three-country field study. J Clin Oncol 1996;14:2756-68.

29 Gujral S, Conroy T, Fleissner C, et al. Assessing quality of life in patients with colorectal cancer: an update of the EORTC quality of life questionnaire. Eur J Cancer 2007;43:1564-73.

30 Greimel E, Nordin A, Lanceley A, et al. Psychometric validation of the European organisation for research and treatment of cancer quality of life Questionnaire-Endometrial cancer module (EORTC QLQEN24). Eur J Cancer 2011;47:183-90.

31 Bjordal K, Ahlner-Elmqvist M, Tollesson E, et al. Development of a European organization for research and treatment of cancer (EORTC) questionnaire module to be used in quality of life assessments in head and neck cancer patients. EORTC quality of life Study Group. Acta Oncol 1994;33:879-85.

32 Kavadas V, Blazeby JM, Conroy T, et al. Development of an EORTC disease-specific quality of life questionnaire for use in patients with liver metastases from colorectal cancer. Eur J Cancer 2003;39:1259-63.

33 Vickery CW, Blazeby JM, Conroy T, et al. Development of an EORTC disease-specific quality of life module for use in patients with gastric cancer. Eur J Cancer 2001;37:966-71.

34 Bergman B, Aaronson NK, Ahmedzai S, et al. The EORTC QLQ-LC13: a modular supplement to the EORTC core quality of life questionnaire (QLQ-C30) for use in lung cancer clinical trials. EORTC Study Group on quality of life. Eur J Cancer 1994;30A:635-42.

35 Blazeby JM, Conroy T, Hammerlid E, et al. Clinical and psychometric validation of an EORTC questionnaire module, the EORTC QLQOES18, to assess quality of life in patients with oesophageal cancer. Eur J Cancer 2003;39:1384-94.

36 Lagergren P, Fayers P, Conroy T, et al. Clinical and psychometric validation of a questionnaire module, the EORTC QLQ-OG25, to assess health-related quality of life in patients with cancer of the oesophagus, the oesophago-gastric junction and the stomach. Eur $J$ Cancer 2007;43:2066-73.

37 Greimel E, Bottomley A, Cull A, et al. An international field study of the reliability and validity of a disease-specific questionnaire module (the QLQ-OV28) in assessing the quality of life of patients with ovarian cancer. Eur J Cancer 2003;39:1402-8.

38 van Andel G, Bottomley A, Fosså SD, et al. An international field study of the EORTC QLQ-PR25: a questionnaire for assessing the health-related quality of life of patients with prostate cancer. Eur $J$ Cancer 2008;44:2418-24.

39 Veritas Health Innovation, Melbourne A. Covidence systematic review software [Internet]. Available: www.covidence.org [Accessed 16 Jan 2020].

40 Higgins JPT, Thomas J, Chandler J, eds. Cochrane Handbook for Systematic Reviews of Interventions version 6.0 (updated July 2019) [Interne]. Cochrane, 2019. www.training.cochrane.org/ handbook

41 Sterne JA, Hernán MA, Reeves BC, et al. ROBINS-I: a tool for assessing risk of bias in non-randomised studies of interventions. BMJ 2016;355:i4919.

42 Whiting PF, Rutjes AWS, Westwood ME, et al. QUADAS-2: a revised tool for the quality assessment of diagnostic accuracy studies. Ann Intern Med 2011;155:529-36.

43 Shea BJ, Reeves BC, Wells G, et al. AMSTAR 2: a critical appraisal tool for systematic reviews that include randomised or nonrandomised studies of healthcare interventions, or both. BMJ 2017;358:j4008.

44 Moher D, Liberati A, Tetzlaff J, et al. Preferred reporting items for systematic reviews and meta-analyses: the PRISMA statement. PLoS Med 2009;6:e1000097.

45 Thuesen J, Rossau HK, Mikkelsen SF, et al. Kræftrehabilitering i Danmark. Kortlægning af praksis på hospitaler og i kommuner. [Cancer rehabilitation in Denmark. Mapping of practice in hospitals and municipalities]. REHPA. Denmark: The Danish Knowledge Centre for Rehabilitation and Palliative Care; Nyborg, 2017. 